\title{
Collapse of cycloidal electron flows induced by misalignments in a magnetically insulated diode
}

\author{
A. L. Garner, Y. Y. Lau, and D. Chernin ${ }^{\text {a) }}$ \\ Department of Nuclear Engineering and Radiological Sciences, University of Michigan, Ann Arbor, \\ Michigan 48109-2104
}

(Received 5 August 1997; accepted 6 March 1998)

\begin{abstract}
The effect of a slight misalignment in the magnetic field on a magnetically insulated diode is investigated. It is found that a slight tilt in the magnetic field, with a minute component along the dc electric field, completely destabilizes the cycloidal electron flow in the crossed-field gap. The final state consists of the classical Brillouin flow superimposed by a turbulent background, together with a slow electron drift across the gap. This disruption of the cycloidal flow is quite insensitive to the emission current density, and is due to the accumulation of space charge in the gap caused by the magnetic misalignment. This result was obtained from a one-dimensional simulation code. It reinforces the notion that the turbulent, near Brillouin-like states are generic in ALL vacuum crossed-field devices. (C) 1998 American Institute of Physics. [S1070-664X(98)02506-3]
\end{abstract}

\section{INTRODUCTION}

When electrons are emitted from a cathode into a vacuum gap with an applied dc voltage and a transverse magnetic field $B>B_{\mathrm{H}}$, with $B_{\mathrm{H}}$ the Hull cutoff magnetic field, ${ }^{1}$ a steady state may be established in which each electron executes a simple, cycloidal orbit and returns to the cathode with the same energy with which it was emitted. ${ }^{2}$ A steady state of this form can be established if the emitted current is below a critical value, $J_{c}$, which depends on the gap voltage, gap spacing, and magnetic field. ${ }^{3}$ This steady state of cycloidal electron flow was found to be very unstable and can be disrupted by a small rf voltage, ${ }^{4}$ or by a small resistance in the external circuit. ${ }^{2}$ The end result is a nearBrillouin $(\mathrm{nB})$ state, which consists of the classical Brillouin flow $^{5}$ superimposed by a turbulent background. ${ }^{2-4}$ This turbulent background consists of low level fluctuations in density and in velocity fields about the mean Brillouin flow. The underlying reason for the cycloidal flow disruption is that electrons emitted from the cathode fail to return to the cathode when a small perturbation is present, thereby leading to a significant increase in the space charge within the gap. Roughly speaking, the maximum amount of charge that can be held within a gap is $C V$, where $C$ is the gap's capacitance in vacuum and $V$ is the gap voltage. ${ }^{6}$ Another way to interpret this ready collapse of cycloidal flows into Brillouin flows is the higher potential energy in the former, for the same gap voltage, the same magnetic field, and the same circulating current in the $\mathbf{E} \times \mathbf{B}$ direction. Note that electrons, in either the cycloidal flow or the Brillouin flow, have zero total energy and zero canonical momentum $P_{y}$ in the $\mathbf{E} \times \mathbf{B}$ direction if they are emitted at rest from the cathode.

A few words on the collapse of the cycloidal flows to Brillouin flows are in order. As the charge density builds up, the cycloidal orbits of the newly entering particles become increasingly flatter due to the presence of the electric field

${ }^{a)}$ Science Applications International Corporation, McLean, Virginia 22102. gradient produced by the space charge of the earlier particles. These flattened cycloidal orbits are sometimes called Slater orbits. ${ }^{7}$ Eventually, and inevitably, Brillouin density is (approximately) reached, the electric field at the cathode surface is depressed to near zero, and the orbits of the newly entering particles, and therefore the whole flow, become (nearly) laminar. This is what we have called near-Brilloiun flow. As we have shown, this state is attained very generally, being produced by almost any perturbation that prevents the initially injected electrons from returning to the cathode. In this state, as in all states leading to it, the canonical momentum $P_{y}$ is exactly constant $(=0)$ for all particles, at least in our one-dimensional (1-D) calculation. The total energy is not conserved during the transient buildup, and fluctuates about a mean in the final state.

In this paper, we investigate the effect of a misalignment in the magnetic field on the otherwise perfect crossed-field geometry. We assume that this misalignment introduces a (small) component in the magnetic field parallel to the dc electric field. This problem is of fundamental interest to the study of magnetic insulation because, in practice, field misalignments are mostly unavoidable. This problem is also of interest in understanding the noise in magnetron injection guns, in which the electric field on the cathode surface may not be orthogonal to the external magnetic field. In fact, it is this concern of the noise problem in gyrotron-driven millimeter wave radars (which employ magnetron injection guns) that motivated the present study. There are other recent studies of cycloidal flow in a crossed-field gap. ${ }^{8-11}$ While singleparticle orbits have been found unstable in nonorthogonal $\mathbf{E}$ and $\mathbf{B}$ due to a gradient in $\mathbf{E},{ }^{12}$ the collapse of the cycloidal flow analyzed in the present paper is due to a very different mechanism.

Note that as long as $\mathbf{E}$ and $\mathbf{B}$ are nonorthogonal, the diode is no longer insulated magnetically. An electron released from the cathode with zero velocity will not return to 
the cathode after one cycloidal orbit. Instead, it will acquire a small drift velocity toward the anode, with the result that this electron will execute multiple cycloidal orbits before it eventually strikes the anode [see Fig. 3(a) and Eq. (4) below]. Thus, even a small misalignment in the magnetic field will allow the continual buildup of space charge and lead to disruption of the flow as described above, even at a low injection current. Since the amount of charge present in a gap, at any instant, depends on the rate at which electrons are released from the cathode and on the rate at which the electrons are depleted to the anode (as a result of field misalignment), the stability property can be quite complicated. In fact, our preliminary study uncovers new stability bands for intermediate values of magnetic field misalignments. These stability bands exhibit a new relaxation-oscillation state that is different from the time-independent state and the $\mathrm{nB}$ state. They remain to be fully characterized.

Let $\psi$ be the angle between the dc magnetic field and the dc electric field. In the perfectly crossed-field (PCF) geometry, $\psi=90^{\circ}$. Misalignment corresponds to $\psi$ less than but close to $90^{\circ}$. The other limit, $\psi=0^{\circ}$, corresponds to the immerse flow in which the electric field and magnetic field are parallel. In this paper we address the stability of electron flow originating from the cathode surface for general values of $\psi$, with special attention to the cases $\psi \approx 90^{\circ}$. We use the one-dimensional, electrostatic simulation code, PDP $1,{ }^{13}$ supplemented by analytic calculations.

The simulations show that the electrons always travel across the gap regardless of the magnetic field when $\psi$ $\neq 90^{\circ}$. Thus, as explained above, there is no magnetic insulation, or Hull cutoff, for nonorthogonal magnetic and electric fields. Tilting the magnetic field by as little as $1^{\circ}$ from the PCF case leads to an instability at very low current densities, sometimes as low as $0.02 J_{\mathrm{CL}}$, where $J_{\mathrm{CL}}$ is the ChildLangmuir limiting current associated with a nonmagnetized diode. ${ }^{14,15}$ For a sufficiently strong current density, this instability is similar to the near-Brillouin flow studied earlier, except that the electrons now travel across the gap with an average velocity $\left(\left\langle v_{x}\right\rangle\right)$ that increases across the gap. A slightly larger tilt $\left(81^{\circ}<\psi<87^{\circ}\right)$ results in a different type of stability characteristic. The phase space is characterized by loops, whose number depends on $\psi$, the magnetic field, and the emission current [see Fig. 3(a)]. As the emitted current is increased from a low value, the number of loops changes, and the amount of charge residing in the gap also changes. This results in stability bands as the injection current is raised from a low value. For $\psi<81^{\circ}$, such stability bands seem to disappear. This case, together with the case $B<B_{\mathrm{H}}$, will be briefly discussed toward the end of this paper. In the Appendix, we outline the analytic derivation of the critical current density, including an oblique magnetic field, under the assumption that all emitted electrons travel to the anode without velocity reversal. A comparison with simulation results is also given there.

\section{MODEL AND SIMULATION RESULTS}

The one-dimensional model used in the simulations is shown in Fig. 1. In this model, the external electric and mag-

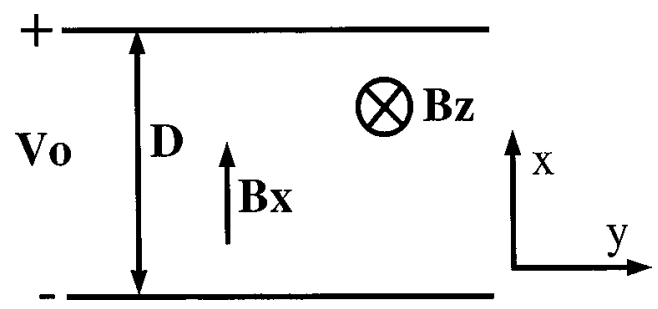

FIG. 1. The model. Electrons are emitted from the cathode at $x=0$ at a constant current density, $J$.

netic fields are given by

$$
\mathbf{E}=\hat{x} E_{x}=-\hat{x} \frac{V_{0}}{D}
$$

and

$$
\mathbf{B}=\hat{x} B_{x}+\hat{z} B_{z}=B(\hat{x} \cos \psi+\hat{z} \sin \psi) .
$$

We fix the anode-cathode separation $D=0.00216 \mathrm{~m}$, electrode area $A=0.003122 \mathrm{~m}^{2}$, and the gap voltage $V_{0}$ $=12000 \mathrm{~V}$. This voltage gives the Hull cutoff magnetic field $B_{\mathrm{H}}=\left(2 m V_{0} / e D^{2}\right)^{1 / 2}=0.171 \mathrm{~T}$ for a PCF geometry. The magnitude of the magnetic field, $B$, and the angle, $\psi$, are varied in the simulations.

At time $t=0$, electrons are injected into the gap at a constant rate with a constant current density $J$, and the gap voltage, $V_{g}(t)$, begins to ramp from zero to its equilibrium value $V_{0}$ as current flows through the external circuit. The cathode potential is held at zero volts while the anode potential is allowed to vary. The electrons are all emitted at a single velocity $u_{0}$ normal to the cathode. The electron emission energy is $0.5 \mathrm{eV}$, which implies a critical current density, $J_{c}=229800 \mathrm{~A} / \mathrm{m}^{2}$, or critical current $I_{c}=A J_{c}=717 \mathrm{~A}$, for the PCF case with $B=1.4 B_{\mathrm{H}}=0.2394 \mathrm{~T}$. ${ }^{3,6}$ For the case where there is no magnetic field, or the magnetic field is parallel to the electric field (i.e., $\psi=0^{\circ}$ ), the maximum electron current density allowed for the time-independent flow solution, $J_{\mathrm{CL}}$, is given by the Child-Langmuir Law, ${ }^{14,15}$

$$
J_{\mathrm{CL}}=\frac{4 \epsilon_{0}}{9 D^{2}}\left(\frac{2 e}{m}\right)^{1 / 2} V^{3 / 2},
$$

where $e$ and $m$ are the charge and mass of the emitted particle (electron), respectively, and $\epsilon_{0}$ is the free space permittivity. For this geometry, $J_{\mathrm{CL}}=658000 \mathrm{~A} / \mathrm{m}^{2}$, or $I_{\mathrm{CL}}=2054 \mathrm{~A}$.

Simulation results for various values of $\psi$ are now described.

First, for $\psi<90^{\circ}$ and $B>B_{\mathrm{H}}$, simulations show that the electrons always travel across the gap, regardless of the magnetic field, current, or specific value of $\psi$. This is simply a reflection of the fact that the gap cannot be magnetically insulated if there is a component of the magnetic field parallel to the electric field. In terms of a single-particle orbit, the velocity of the electron as a function of time can be written as 


$$
\begin{aligned}
\mathbf{v}(t)= & \mathbf{a}_{\|} t+\frac{\mathbf{E} \times \mathbf{B}}{|\mathbf{B}|^{2}}+\left|\frac{\mathbf{E} \times \mathbf{B}}{|\mathbf{B}|^{2}}\right|(\sin \psi \sin \Omega t, \\
& -\cos \Omega t,-\cos \psi \sin \Omega t),
\end{aligned}
$$

where $\Omega=|e||B| / m$ is the electron cyclotron frequency and $\mathbf{a}_{\|}$is the acceleration parallel to the electric field, given by

$$
\mathbf{a}_{\|}=\frac{|e| V}{m D} \cos \psi(\cos \psi, 0, \sin \psi) .
$$

The first term in Eq. (4) represents acceleration along the magnetic field line. It clearly shows that the $x$ coordinate will eventually reach the anode $x=D$, except in the PCF case $\psi=90^{\circ}$. The second term of Eq. (4) represents the $\mathbf{E} \times \mathbf{B}$ drift and the last term of (4) represents the cycloidal motion.

What is more surprising is that a small departure from the PCF case leads to a complete breakdown of the cycloidal flow, even at a very low level of the emission current density. The result for the case $\psi=89^{\circ}$ is shown in Figs. 2(a)2(d) where $B=1.4 B_{\mathrm{H}}, J=0.08 J_{\mathrm{CL}}=0.24 J_{c}$. (Recall that for the PCF case, $\psi=90^{\circ}$, the cycloidal flow remains stable for $J$ up to $\left.J_{c}\right)^{3}$

At $t=0.263 \mathrm{~ns}$, the electrons have completed fewer than two cycles [Fig. 2(a)], and the electron orbits are similar to the initial state for the PCF $\left(\psi=90^{\circ}\right) .{ }^{2-4,9}$ Note that the time it takes for the electrons emitted from the cathode to return to the cathode is approximately equal to the inverse of the gyrofrequency $(2 \pi / \Omega=0.149 \mathrm{~ns})$. After approximately one nanosecond, the phase space no longer resembles a simple ellipse [Fig. 2(b)]. The electron paths have begun to move closer to the anode at this point. The disconnected phase space trajectories shown in Fig. 2(b) arise from certain segments of the electron flow returning to the cathode. After approximately $6.85 \mathrm{~ns}$, the electron flow has begun to collapse [Fig. 2(c)], quite similar to the development toward the turbulent state of the PCF case. ${ }^{2-4}$ Note that some of the electrons have traveled across the gap and that $\left\langle v_{x}\right\rangle$ is close to zero over part of the gap, but increases closer to the anode. After approximately ten nanoseconds, the electron flow has completely collapsed [Fig. 2(d)]. Note that the electrons near the anode are not as tightly grouped together as those near the cathode. Increasing the current causes the electrons to collapse more rapidly and more closely together, although the basic shape and characteristic of the collapse are the same.

For comparison, for $\psi=90^{\circ}$ and $B=1.4 B_{\mathrm{H}}$, the cycloidal flow remains stable for all injection current density up to $J_{c}=0.33 J_{\mathrm{CL}}$.

The fact that a small tilt in the magnetic field (from $\psi$ $=90^{\circ}$ to $89^{\circ}$ ) can completely destabilize the flow at a substantially reduced emission current may be understood by referring to Eq. (4) and Fig. 2(b). Equation (4) and the $x$ component of Eq. (5) suggest that an electron is accelerated toward the anode whenever $\psi<90^{\circ}$. Thus, this electron may fail to return to the cathode upon completion of the cycloid, as shown in Fig. 2(b). As a result, charge continues to build up in the gap, leading to the breakdown of the cycloidal flow into the $\mathrm{nB}$ state. This scenario is revealed in our simulations; namely, the total charge within the gap builds up
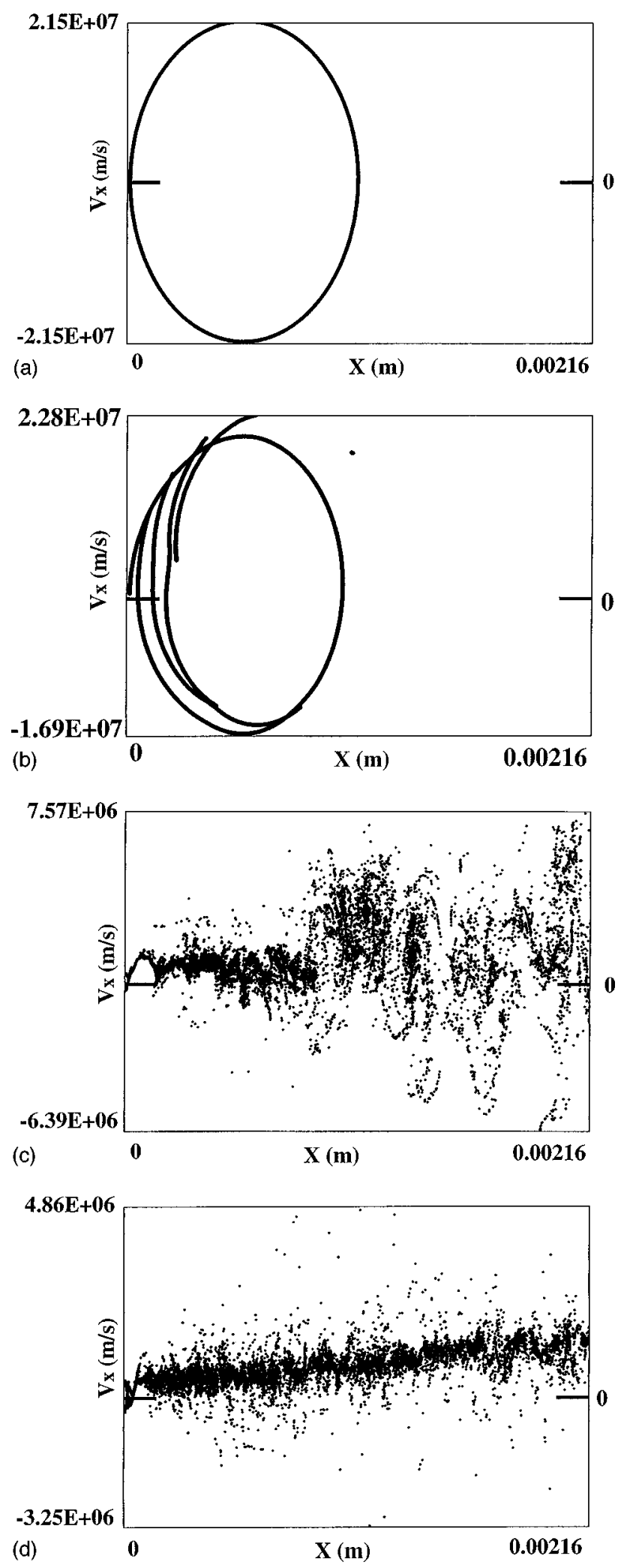

FIG. 2. The phase space plots (a) after one cycloidal orbit, (b) after four orbits, (c) approaching the final near-Brillouin state after approximately 7 $\mathrm{ns}$, and (d) after reaching the final near-Brillouin state after approximately $10 \mathrm{~ns}$.

roughly linearly in time (instead of exponentially in time), almost up to the breakdown of the cycloidal flow.

The above scenario suggests that the collapse of the cycloidal flow depends on the accumulation of space charge in 


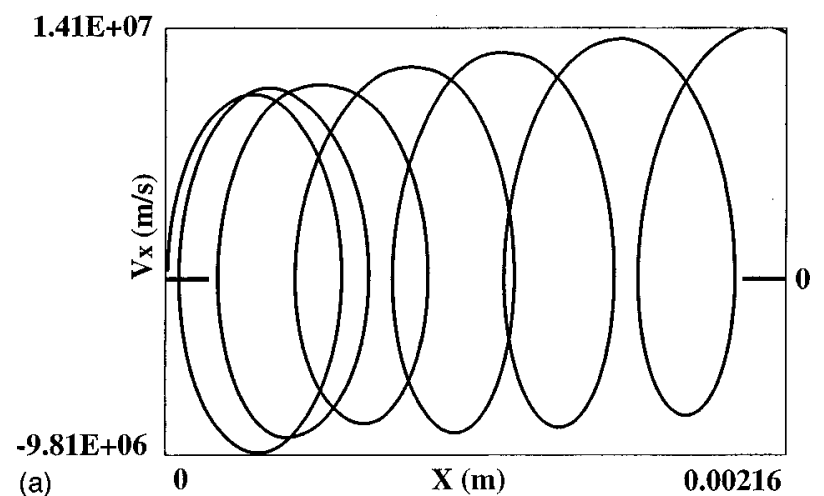

(a)

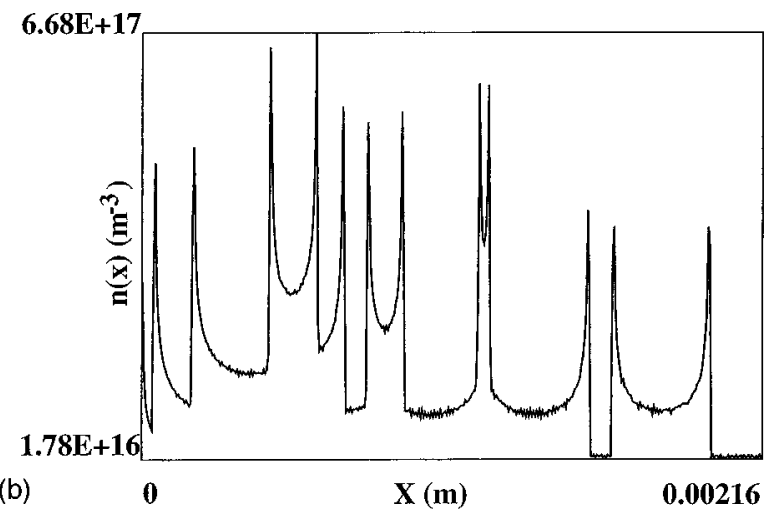

FIG. 3. (a) The phase space plot for a stable electron flow when $\psi=87^{\circ}$, $B=1.4 B_{\mathrm{H}}, J=0.07 J_{\mathrm{CL}}$. (b) The density plot for this stable flow. Note the density spikes where the electrons reverse direction in $x$ (i.e., $v_{x}=0$ ).

the gap. This accumulation depends on the emission rate and the rate at which electrons are depleted to the anode as a result of $a_{x}$, the acceleration in the $x$ direction.

Figure 3 shows the case where a time-independent (but complicated) flow can be maintained if the injection current density is sufficiently low. In this figure, we set $\psi=87^{\circ}, B$ $=1.4 B_{\mathrm{H}}$, and $J=0.07 J_{\mathrm{CL}}=0.21 J_{c}$. The phase space for this stable case is characterized by the formation of several loops in phase space as the electrons travel across the gap. In the process of traversing the gap, the electrons must turn around, or change direction, more than once [Fig. 3(a)]. The electron density becomes very large at the locations of the zero $v_{x}$ electrons [Fig. 3(b)]. This suggests that increasing the current can significantly build up the already large amount of charge in the gap and easily cause the flow to collapse into an unstable, $\mathrm{nB}$ flow for $\psi=87^{\circ}, B=1.4 B_{\mathrm{H}}$, and $J$ $=0.10 J_{\mathrm{CL}}=0.30 J_{c}$ (Fig. 4). This collapse occurs at significantly lower current densities than the collapse that occurs for the PCF case $\left(\psi=90^{\circ}\right)$ with the same magnetic field ( $J$ $=0.33 J_{\mathrm{CL}}=J_{c}$ ). This sensitivity to $J$ due to zero $v_{x}$ electrons may also be responsible for the instabilities at higher angles as well. Increasing $\psi$ increases the number of loops for a constant $J$ and $B$. If the number of loops becomes too high, or if the number of intersections of electron paths at zero $v_{x}$ becomes large, the gap could contain several regions of high electron density. A very small current would be able to disrupt the flow for such a high electron density. This is verified by simulation for $89^{\circ}<\psi<90^{\circ}$ and all values of $J$ greater than $0.02 J_{C L}$ that were tested. Simply put, for this range $\psi$,

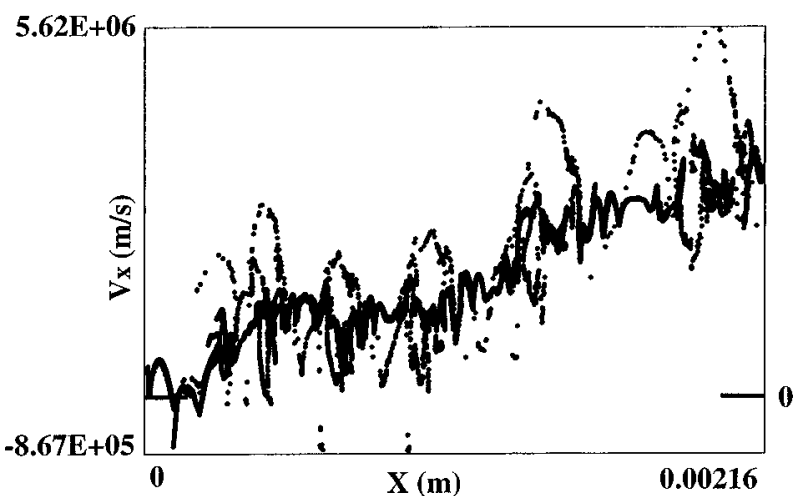

FIG. 4. The phase space plot for the breakdown to the turbulent nearBrillouin flow by sufficiently increasing the current density for $\psi=87^{\circ}, B$ $=1.4 B_{\mathrm{H}}, J=0.10 J_{\mathrm{CL}}$.

the current density required to disrupt the time-independent cycloidal flow is much lower than that for the PCF case $(\psi$ $=90^{\circ}$ ) with the same magnetic field.

Further tilting the magnetic field $\left(81^{\circ}<\psi<87^{\circ}\right)$ changes the stability characteristics of the flow. At a fixed value of $B\left(B=1.4 B_{\mathrm{H}}\right.$, for instance), the cycloidal flow may exhibit alternate bands of stability and instability as the injection current is increased. The underlying reason for this complicated phenomenon for these values of $\psi$ is that fewer loops appear in the phase space, leading to fewer density spikes in the gap and increased electron flow stability for lower currents. As one decreases $\psi$ farther away from $90^{\circ}$, the number of loops continues to decrease. The number of loops that the electrons travel through in phase space also depends on the magnetic field and current density. For a constant $\psi$ and a constant magnetic field $B$, simulation results show that increasing the current causes a timedependent relaxation oscillation state due to the buildup of charge near the anode when the current density $J$ reaches approximately $0.10 J_{\mathrm{CL}}$. This instability results from electrons slowing down to nearly zero $v_{x}$ near the anode, leading to an electron density spike. This bunching of negative charge pushes the electrons away from the anode and causes them to attempt to form another loop. However, as this loop forms, charge builds up farther in the gap and pushes the electrons back to the anode, separating the electrons forming the additional loop from the main body of the electrons. This process is periodic and leads to a relaxation oscillation state for $J$ on the order of $0.10 J_{\mathrm{CL}}$. Slightly increasing the current above the range for this instability band leads to stable flow with one fewer loop in phase space than the previous band of stable flow for the lower currents. A higher injection current can lead to a stable flow by causing the electrons near the anode to speed up sufficiently to lower the density at the anode, minimizing the charge buildup at the anode. This results in the formation of bands that exhibit time-independent flow for these values of $\psi$, with the number of bands limited by the number of loops in the phase space. For example, for $B=1.4 B_{\mathrm{H}}$ and $\psi=85^{\circ}$, the cycloidal flow is stable for 0 $<J / J_{\mathrm{CL}}<0.04$ and $0.10<J / J_{\mathrm{CL}}<0.12$, but is unstable for $0.05<J / J_{\mathrm{CL}}<0.09$. Increasing the current density beyond 
$0.12 J_{\mathrm{CL}}\left(0.4 J_{c}\right)$ causes the flow to collapse into the nearBrillouin state.

Our preliminary study of the stability bands does not indicate any trend on the total charge within the gap. For example, for the case $B=1.4 B_{\mathrm{H}}, \psi=85^{\circ}$ reported above, the total charge per unit area, $Q / A$ in $\mathrm{C} / \mathrm{m}^{2}$, equals $(-4.03$ $\left.\times 10^{-5},-2.13 \times 10^{-5}\right)$ for the time-independent states with $J / J_{\mathrm{CL}}=(0.039,0.11)$; while $Q / A=\left(-3.77 \times 10^{-5},-2.75\right.$ $\times 10^{-5}$ ) for the relaxation-oscillation states with $J / J_{\mathrm{CL}}$ $=(0.051,0.089)$.

Narrower stability bands, which do not involve loop formation in phase space, have also been found for currents around $0.30 J_{\mathrm{CL}}$ for various combinations of $B$ and $\psi$. For example, a narrow stability band exists for $B=1.25 B_{\mathrm{H}}, \psi$ $=81.5^{\circ}$, and $J \approx 0.27 J_{\mathrm{CL}}$. These preliminary results indicate that finding a current that leads to an instability does not imply that the flow is unstable at higher currents.

For $\psi<70^{\circ}$, an instability characterized by the periodic emission of a high-density electron blob can be observed through simulations for $B>B_{\mathrm{H}}$. This mechanism is actually quite similar to that observed for $\psi=90^{\circ}$ and $B<B_{\mathrm{H}}{ }^{6}$ (i.e., for a noninsulated, PCF diode). The phase space is characterized by a straight line of electrons for low values of $B$, which becomes more curved as the magnetic field is increased. If the magnetic field or $\psi$ is increased sufficiently, a loop or loops can form in the phase space, changing the mechanism of instability. A theory based on a model of an electron emitted from the cathode traveling across the gap without encountering $v_{x}=0$ yields critical currents, in excellent agreement with those obtained through simulation for $\psi<70^{\circ}$ for all cases where the electrons travel across the gap without turning around. See the Appendix and Fig. 5 for details.

Finally, for all values of $\psi$, the phase space for $B<B_{\mathrm{H}}$ (including the previously studied noninsulating PCF case ${ }^{6}$ ) is characterized by a straight line for low $B$, but becomes more curved as the magnetic field is increased to $B_{\mathrm{H}}$. The instability in this case is characterized by a high-density electron blob that is emitted periodically from the cathode and travels across the gap. ${ }^{6}$

\section{CONCLUSION}

The simulation results presented above show that a slight misalignment of the fields in a crossed-field device could strongly destabilize the cycloidal flow. A misalignment as small as $1^{\circ}$ can collapse the cycloidal flow to a turbulent near-Brillouin state, even for a very low injection current. This further supports the statement made previously that any small perturbation in a crossed-field geometry, such as the application of a small $\mathrm{rf}$ voltage ${ }^{4}$ or the presence of a small external resistance, ${ }^{2}$ collapses the cycloidal flow. The primary differences, though not critical for flow stability, between the misalignment and the perturbations studied earlier are that the gap can no longer be magnetically insulated and $\left\langle v_{x}\right\rangle$ is no longer zero over the entire gap, but increases toward the anode. Also interesting, although not yet fully characterized, is the presence of instability bands for larger misalignments on the order of a few degrees. These stability bands were found both for low currents $\left(J \approx 0.10 J_{\mathrm{CL}}\right)$ and for higher currents $\left(J \approx 0.30 J_{\mathrm{CL}}\right)$.

\section{ACKNOWLEDGMENTS}

This work was supported by the Department of Navy Grant No. N 00014-97-1-G001 issued by the Naval Research Laboratory, by the Department of Defense Augmentation Awards for Science and Engineering Training, and by the Naval Surface Warfare Center/Crane Division.

\section{APPENDIX: DERIVATION OF CRITICAL CURRENT, ASSUMING NO VELOCITY REVERSAL IN THE ELECTRON ORBITS}

In this appendix, we analytically determine the maximum current for time-independent electron flows in an oblique electric and magnetic field. The crucial assumption is introduced, namely, all electrons, emitted at rest from the cathode, travel directly to the anode without velocity reversal. This assumption enables us to use the Llewellyn formulation and to adopt the condition of the zero electric field on the cathode surface to determine the critical current density. ${ }^{3,15,16}$ Since this appendix is a straightforward generalization of Ref. 16 to an oblique magnetic field, we simply state the results without giving the details. Comparison with simulation results is shown in Fig. 5 and discussed further below.

We first define the normalized transit time, $T$, as

$$
T=\Omega t_{f},
$$

where $t_{f}$ is the time of flight of an electron to the anode. The Llewellyn formulation, which is a Lagrangian formulation for time-independent laminar electron flows, ${ }^{15,16}$ gives the following expression for the gap spacing $D$ in terms of $T$ :

$$
D=\frac{|e| J}{m \epsilon_{0}} \frac{1}{\Omega^{3}}\left(\cos ^{2} \psi \frac{T^{3}}{6}+\sin ^{2} \psi(T-\sin T)\right),
$$

where, as in the main text, $J$ is the injection current density, $\psi$ is the angle between the magnetic and electric field, and the other familiar symbols have been defined in the main text. Equation (A2) can be rewritten as

$$
\frac{1}{f(T)}=\frac{|e| J}{m \epsilon_{0} \Omega^{3} D},
$$

where $f(T)$ is given by

$$
f(T)=\cos ^{2} \psi \frac{T^{3}}{6}+\sin ^{2} \psi(T-\sin T) .
$$

From conservation of energy at $t=t_{f}$, we find

$$
|e| V=\frac{1}{2} m\left(\frac{|e| J}{m \epsilon_{0} \Omega^{2}}\right)^{2} g(T),
$$

where $g(T)$ is defined as 

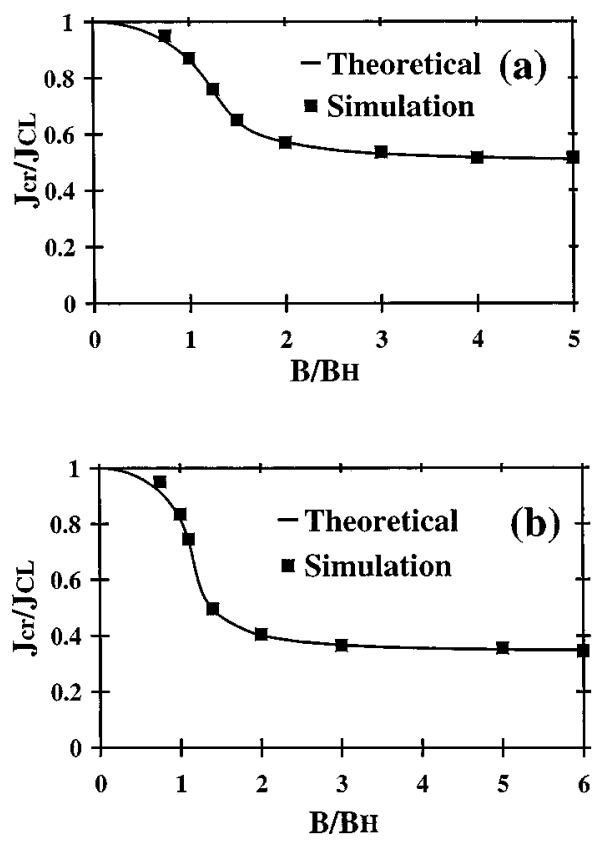
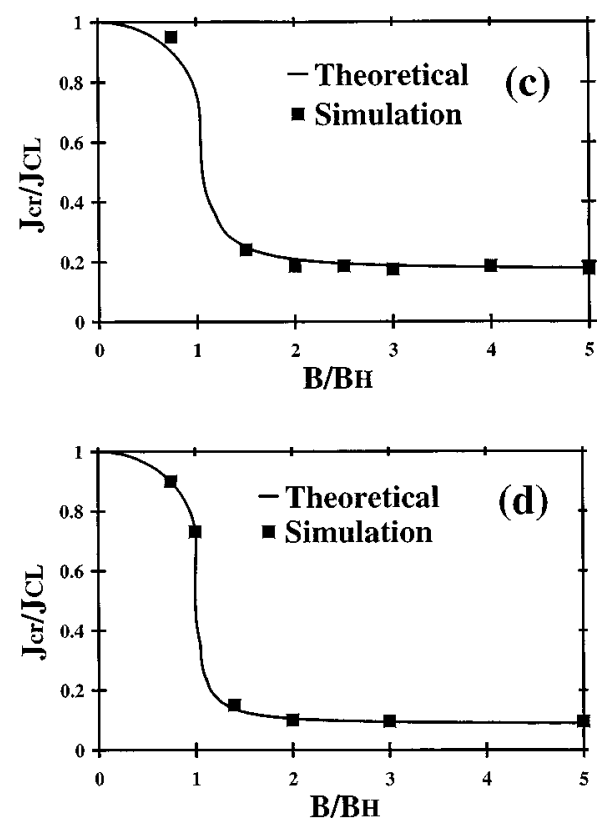

FIG. 5. A comparison of theoretically predicted critical current, normalized to the Child-Langmuir value, as a function of the magnetic field, normalized to the Hull cutoff, for (a) $\psi=60^{\circ}$, (b) $\psi=70^{\circ}$, (c) $\psi=80^{\circ}$, and (d) $\psi=85^{\circ}$.

$$
\begin{aligned}
g(T) \equiv & \left(\cos ^{2} \psi \frac{T^{2}}{2}+\sin ^{2} \psi(1-\cos T)\right)^{2}+[\sin \psi(T \\
& -\sin T)]^{2}+\left(\cos \psi \sin \psi\left(\frac{T^{2}}{2}-1+\cos T\right)\right)^{2}
\end{aligned}
$$

We next define normalized current and voltage as ${ }^{16}$

$$
\widetilde{J} \equiv \frac{J}{m \epsilon_{0} \Omega^{3} D /|e|}
$$

and

$$
\tilde{V} \equiv \frac{V}{m \Omega^{2} D^{2} /|e|},
$$

respectively. Equation (A7b) can be rewritten as

$$
\tilde{V}=\frac{1}{2}\left(\frac{B_{\mathrm{H}}}{|\mathbf{B}|}\right)^{2},
$$

where $B_{\mathrm{H}}$ is the Hull cutoff magnetic field defined earlier as $\left(2 m V / e D^{2}\right)^{1 / 2}$ for zero injection velocity. Equation (A7a) permits us to directly rewrite Eq. (A3) as

$$
\tilde{J}_{\mathrm{cr}}=\frac{1}{f(T)},
$$

which gives the expression for the normalized critical current as a function of normalized time with $f(T)$ given by Eq. (A4). Similarly, Eqs. (A7a), (A7b), and (A8) can be applied to Eq. (A5) to obtain

$$
\tilde{V}=\frac{1}{2} \frac{g(T)}{f^{2}(T)},
$$

which gives the expression for the normalized voltage as a function of normalized time with $f(T)$ given by Eq. (A4) and $g(T)$ given by Eq. (A6). Using Eq. (A7c), Eq. (A9) can be rewritten as

$$
\frac{|B|}{B_{\mathrm{H}}}=\frac{f(T)}{\sqrt{g(T)}} .
$$

Equations (A8) and (A10) give expressions for the normalized critical current, $J_{\mathrm{cr}}$, and the magnetic field normalized to $B_{\mathrm{H}}$ parametrically through the normalized transit time $T$ for arbitrary angle $\psi$. It can be shown that in the limit $\psi$ $=0^{\circ}$, we obtain a normalized critical current of $\widetilde{J}_{\mathrm{cr}} / \widetilde{J}_{\mathrm{CL}}$ $=1$, which is simply the well-known Child-Langmuir condition for an immersed flow. In the limit $\psi=90^{\circ}$, we recover the results ${ }^{16}$ for the crossed-field case for $B<B_{\mathrm{H}}$. One can also show that $\tilde{J}_{\mathrm{cr}} / \tilde{J}_{\mathrm{CL}}$ approaches $\cos \psi$ as $B \rightarrow \infty$ from the equations given here (also see Fig. 5).

Figures 5(a)-5(d) show plots of the normalized critical current versus the normalized magnetic field predicted theoretically from Eqs. (A8) and (A10) and observed by simulation for $\psi=60^{\circ}, 70^{\circ}, 80^{\circ}$, and $85^{\circ}$. The theory agrees very well with simulation results for $\psi<80^{\circ}$ for all values of $B$ [Figs. 5(a) and 5(b)]. In these cases, the electrons usually do not turn around as they traverse the gap, as required by the Lewellyn formulation. In general, for these values of $\psi$, the electrons may be decelerated in $v_{x}$ as they cross the gap, but they do not change directions. In the cases where the electrons do turn around, they turn around near the cathode, but otherwise satisfy the assumption of no velocity reversal, thus still leading to excellent agreement between the theoretical and simulation results. This is also observed as $\psi$ is increased to $\psi \geqslant 80^{\circ}$, where theory and simulation are in excellent agreement for high $B\left(B>2 B_{\mathrm{H}}\right)$ and low $B\left(B<B_{\mathrm{H}}\right)$ [Fig. 
5(c)]. For intermediate $B\left(B_{\mathrm{H}}<B<2 B_{\mathrm{H}}\right)$, the low-current stability bands discussed in the main text do occur. The theory fails to predict these bands. Since these bands contain many electrons changing directions, they fail to fulfill the assumption of no velocity reversal, and one might anticipate that the theory would fail to accurately predict the maximum current for time-independent flow. However, increasing the current beyond the stability bands can cause the electron to travel across the gap without turning around (or turning around only very close to the cathode) in some cases, thus satisfying that assumption [Fig. 5(d)]. The predicted critical currents agree very well for high magnetic fields and fairly well for $B<B_{\mathrm{H}}$.

These results [Figs. 5(a) $-5(\mathrm{~d})$ ] indicate that the critical current does approach a constant (or at least a fairly constant value) as the magnetic field becomes very large. This indicates that one can reach a point where increasing the magnetic field has virtually no effect on the critical current.

${ }^{1}$ A. W. Hull, Phys. Rev. 18, 31 (1921).

${ }^{2}$ P. J. Christenson, D. P. Chernin, A. L. Garner, and Y. Y. Lau, Phys. Plasmas 3, 4455 (1996).

${ }^{3}$ P. J. Christenson and Y. Y. Lau, Phys. Plasmas 1, 3725 (1994); 3, 4293 (1996).
${ }^{4}$ P. J. Christenson and Y. Y. Lau, Phys. Rev. Lett. 76, 3324 (1996).

${ }^{5}$ L. Brillouin, Phys. Rev. 67, 260 (1945).

${ }^{6}$ P. J. Christenson, Ph.D. thesis, University of Michigan, Ann Arbor, 1996.

${ }^{7}$ J. C. Slater, Microwave Electronics (Van Nostrand, New York, 1950), p. 340.

${ }^{8}$ J. P. Verboncoeur and C. K. Birdsall, Phys. Plasmas 3, 712 (1996).

${ }^{9}$ V. P. Gopinath, J. P. Verboncoeur, and C. K. Birdsall, Phys. Plasmas 3, 2766 (1996).

${ }^{10}$ J. Swegle, IEEE Trans. Plasma Sci. PS-24, 1277 (1996).

${ }^{11}$ B. H. Vanderberg and J. E. Eninger, Phys. Plasmas 4, 256 (1997).

${ }^{12}$ S. Riyopoulos, Phys. Plasmas 3, 4355 (1996).

${ }^{13}$ PDP1 (Plasma Device Planar 1 Dimensional), (C) 1990-1993 Regents of the University of California, Plasma Theory and Simulation Group, Berkeley, CA. Available from the Software Distribution Office of ILP, 205 Cory Hall, Berkeley, CA 94720; electronic mail: software@eecs. berkeley.edu. This is a one-dimensional code that includes all three components of velocity. See, also, J. P. Verboncoeur, M. V. Alves, V. Vahedi, and C. K. Birdsall, J. Comput. Phys. 131, 321 (1993).

${ }^{14}$ C. D. Child, Phys. Rev. 32, 492 (1911) I. Langmuir, ibid. 21, 419 (1923).

${ }^{15}$ C. K. Birdsall and W. B. Bridges, Electron Dynamics of Diode Regions (Academic, New York, 1966); R. B. Miller, An Introduction to the Physics of Intense Charged Particle Beams (Plenum, New York, 1982); R. C. Davidson, Physics of Nonneutral Plasmas (Addison-Wesley, Redwood City, CA, 1990).

${ }^{16}$ Y. Y. Lau, P. J. Christenson, and D. Chernin, Phys. Fluids B 5, 4486 (1993). 